\title{
Key Role of the Oxidized Citrate Free Radical in the Nucleation Mechanism of the Metal Nanoparticles Turkevich Synthesis
}

Sarah Al Gharib, ${ }^{1,2}$, Jean-Louis Marignier, ${ }^{1}$ Abdel Karim El Omar, ${ }^{2}$ Adnan Naja, ${ }^{2}$ Sophie Le Caer, ${ }^{3}$ Mehran Mostafavi, ${ }^{1}$ and Jacqueline Belloni ${ }^{1 *}$

${ }^{1}$ Laboratoire de Chimie-Physique/ELYSE, UMR 8000 CNRS/UPS, Univ. Paris Sud, Université Paris-Saclay, Bât. 349, F-91405 Orsay Cedex, France.

${ }^{2}$ Laboratoire de Physique et Modélisation, Université Libanaise, Tripoli, Lebanon.

${ }^{3}$ Laboratoire LIONS, DSM/IRAMIS/NIMBE UMR 3685 CNRS/CEA/Saclay, Université Paris-Saclay, Bât. 546, F-91191 Gif-sur-Yvette, Cedex, France.

Table S1. Radiolytic yields (in $10^{-7}$ mol $\mathrm{J}^{-1}$ units) of final products and mechanism in $\gamma$ irradiated citrate solutions with or without silver ions. The uncertainties on the values are $10 \%$.

\begin{tabular}{|c|c|c|c|c|c|}
\hline Solution & $\begin{array}{c}\mathbf{G}\left(\mathrm{Ag}_{\mathrm{NP}}\right) \\
\mathbf{p H} 8^{a}\end{array}$ & $\begin{array}{c}\text { G(DCA) } \\
\text { pH } 8\end{array}$ & $\begin{array}{c}\text { G(H } \mathbf{H}_{2}{ }^{b} \\
\text { pH 1.6 }\end{array}$ & $\begin{array}{c}\mathrm{G}\left(\mathrm{CO}_{2}\right) \\
\text { pH } 1.6\end{array}$ & $\begin{array}{l}\text { Mechanism } \\
\text { (Figure S4) }\end{array}$ \\
\hline $\begin{array}{c}\text { Cit, Ar-satd expl } \\
\text { calcd }\end{array}$ & 0 & $\begin{array}{l}1.74 \\
1.75\end{array}$ & $\begin{array}{l}1.8 \\
1.82\end{array}$ & $\begin{array}{l}1.8 \\
1.82\end{array}$ & $\begin{array}{l}\mathrm{G}\left(\mathrm{CO}_{2}\right)=\mathrm{G}(\mathrm{DCA})=1 / 2[\mathrm{G}(\mathrm{OH})+ \\
\left.G\left(\mathrm{H}_{2} \mathrm{O}_{2}\right)\right] \\
\mathrm{G}\left(\mathrm{H}_{2}\right)=1 / 2\left[G\left(\mathrm{H}^{*}\right)-G\left(\mathrm{H}_{2} \mathrm{O}_{2}\right)\right]+ \\
G\left(\mathrm{H}_{2}\right)\end{array}$ \\
\hline $\begin{array}{ll}\begin{array}{l}\text { Cit, } \\
\text { expl }\end{array} & \mathrm{Ag}^{+}, \quad \text { Ar-satd } \\
& \text { calcd }\end{array}$ & $\begin{array}{l}6.3 \\
6.2\end{array}$ & $\begin{array}{l}2.4 \\
2.8\end{array}$ & $\begin{array}{l}0.37 \\
0.42\end{array}$ & $\begin{array}{l}2.9 \\
2.9\end{array}$ & $\begin{array}{l}\mathrm{G}\left(\mathrm{Ag}_{\mathrm{NP}}\right)=G\left(e_{a q}{ }^{-}\right)+G\left(H^{*}\right)+G\left(\mathrm{OH}^{*}\right) \\
\mathrm{G}\left(\mathrm{CO}_{2}\right)=\mathrm{G}(\mathrm{DCA})=G\left(\mathrm{OH}^{*}\right) \\
\mathrm{G}\left(\mathrm{H}_{2}\right)=G\left(\mathrm{H}_{2}\right)\end{array}$ \\
\hline $\begin{array}{c}\text { Cit, } \mathrm{N}_{2} \mathrm{O} \text {-satd expl } \\
\text { calcd }\end{array}$ & 0 & $\begin{array}{l}4.3 \\
3.8\end{array}$ & - & - & $\begin{array}{l}\mathrm{G}\left(\mathrm{CO}_{2}\right)=\mathrm{G}(\mathrm{DCA})=1 / 2\left[G\left(e_{a q}{ }^{-}\right)+\right. \\
\left.G\left(\mathrm{OH}^{*}\right)+G\left(\mathrm{H}^{\circ}\right)\right]+G\left(\mathrm{H}_{2} \mathrm{O}_{2}\right) \\
\mathrm{G}\left(\mathrm{H}_{2}\right)=G\left(\mathrm{H}_{2}\right)\end{array}$ \\
\hline $\begin{array}{l}\text { Cit, } \mathrm{Ag}^{+}, \mathrm{N}_{2} \mathrm{O}-\text {-satd } \\
\text { expl } \\
\text { calcd }\end{array}$ & $\begin{array}{l}6.3 \\
6.2\end{array}$ & 5.8 & - & - & $\begin{array}{l}\mathrm{G}\left(\mathrm{Ag}_{\mathrm{NP}}\right)=G\left(e_{a q}^{-}\right)+G\left(H^{*}\right)+G\left(\mathrm{OH}^{*}\right) \\
\mathrm{G}\left(\mathrm{CO}_{2}\right)=\mathrm{G}(\mathrm{DCA})=G\left(e_{a q^{-}}\right)+ \\
G\left(\mathrm{OH}^{*}\right) \\
\mathrm{G}\left(\mathrm{H}_{2}\right)=G\left(\mathrm{H}_{2}\right)\end{array}$ \\
\hline
\end{tabular}

The primary yields $($ at $>100 \mathrm{~ns})$ of radical and molecular products of irradiated water are: ${ }^{29}$

a. At pH 8: $\mathrm{H}_{2} \mathrm{O} \mathrm{vvvv} \rightarrow e_{a q}^{-}$(2.8), $\mathrm{H}^{+}$(2.8), $\mathrm{H}^{*}$ (0.62), $\mathrm{OH}^{*}$ (2.8), $\mathrm{H}_{2}(0.42), \mathrm{H}_{2} \mathrm{O}_{2}(0.73)$

b. At $\mathrm{pH}$ 1.6: $\mathrm{H}_{2} \mathrm{O} v v v v \rightarrow \mathrm{H}^{*}$ (3.52), $\mathrm{OH}^{*}$ (2.9), $\mathrm{H}_{2}(0.42), \mathrm{H}_{2} \mathrm{O}_{2}(0.73)$ 
Table S2. Rate constants of reactions involved in the irradiated silver solutions

\begin{tabular}{|c|c|c|c|}
\hline & Reaction & $\begin{array}{l}\text { Rate constant } \\
\left(\mathbf{M}^{-1} \mathbf{s}^{-1}\right)\end{array}$ & References \\
\hline (1) & $\mathrm{Ag}^{+}+\mathrm{e}_{\mathrm{aq}}^{-} \rightarrow \mathrm{Ag}^{0}$ & $3.6 \times 10^{10}$ & [44] \\
\hline$(2)$ & $\mathrm{Ag}^{+}+\mathrm{H}^{\cdot} \rightarrow \mathrm{Ag}^{0}+\mathrm{H}^{+}$ & $3.1 \times 10^{10}$ & {$[46]$} \\
\hline (3) & $\mathrm{Ag}_{\mathrm{Cit}}+\mathrm{Ag}_{\mathrm{Cit}}^{+} \rightarrow \mathrm{Ag}_{2, \mathrm{Cit}}^{+}$ & & $\begin{array}{l}{[19],[20],} \\
\text { this work }\end{array}$ \\
\hline \multirow[t]{2}{*}{ (4) } & $\mathrm{Ag}_{2, \mathrm{Cit}}{ }^{+}+\mathrm{Ag}_{2, \mathrm{Cit}}{ }^{+} \rightarrow \quad \mathrm{Ag}_{4, \mathrm{Cit}}{ }^{2+}$ & & $\begin{array}{l}{[19],[20],} \\
\text { this work }\end{array}$ \\
\hline & $\mathrm{Ag}_{4, \mathrm{Cit}}^{2+} \rightarrow^{\cdots \cdots} \rightarrow \mathrm{Ag}_{\mathrm{NP}, \mathrm{Cit}}$ & & {$[19]$} \\
\hline (5) & $\begin{aligned} \mathrm{Cit}+\mathrm{OH}^{\cdot} \rightarrow & \mathrm{Cit}-\mathrm{OH}^{*} \rightarrow \\
& \mathrm{Cit}(-\mathrm{H})^{\cdot}+\mathrm{H}_{2} \mathrm{O}\end{aligned}$ & $5.0 \times 10^{7}$ & $\begin{array}{l}{[27],[38],[40]} \\
\text { this work }\end{array}$ \\
\hline (6) & $\mathrm{Cit}+\mathrm{H}^{\cdot} \rightarrow \mathrm{Cit}(-\mathrm{H})^{\bullet}+\mathrm{H}_{2}$ & $4.0 \times 10^{5}$ & {$[39]$} \\
\hline (7) & $\mathrm{Cit}(-\mathrm{H})^{\bullet}+\mathrm{Cit}(-\mathrm{H})^{\bullet} \rightarrow \mathrm{DCA}+\mathrm{CO}_{2}$ & $3.5 \times 10^{6}$ & [27], this work \\
\hline (8) & $\mathrm{N}_{2} \mathrm{O}+\mathrm{e}_{\mathrm{aq}}^{-} \rightarrow \mathrm{N}_{2}+\mathrm{OH}^{-}+\mathrm{OH}^{-}$ & $8.7 \times 10^{9}$ & {$[43]$} \\
\hline (9) & $\mathrm{N}_{2} \mathrm{O}+\mathrm{H}^{\cdot} \rightarrow \quad \mathrm{N}_{2}+\mathrm{OH}^{\bullet}$ & $2.1 \times 10^{6}$ & [42] \\
\hline (10) & $\mathrm{H}_{2} \mathrm{O}_{2}+\mathrm{H}^{\bullet} \rightarrow \mathrm{OH}^{*}+\mathrm{H}_{2} \mathrm{O}$ & $9 \times 10^{7}$ & {$[41]$} \\
\hline (11) & $\mathrm{Ag}_{\text {cit }}{ }^{+}+\mathrm{Cit}(-\mathrm{H})^{\circ} \rightarrow \mathrm{Ag}_{\text {cit }}{ }^{+}-\mathrm{Cit}(-\mathrm{H})^{\circ}$ & & this work \\
\hline (12) & $\begin{array}{r}\mathrm{Ag}_{\mathrm{Cit}}^{+}-\mathrm{Cit}(-\mathrm{H})^{\bullet}+\mathrm{Ag}_{\mathrm{Cit}}^{+} \rightarrow \\
\mathrm{Ag}_{2, \mathrm{Cit}}^{+}+\mathrm{DCA}+\mathrm{CO}_{2}\end{array}$ & $8 \times 10^{4}$ & this work \\
\hline (13) & $\begin{aligned} & \mathrm{Ag}_{2, \mathrm{cit}}{ }^{+}+\mathrm{Cit}(-\mathrm{H})^{\bullet} \rightarrow \\
& \mathrm{Ag}_{2, \mathrm{Cit}}+\mathrm{DCA}+\mathrm{CO}_{2}\end{aligned}$ & & this work \\
\hline (14) & $\begin{aligned} & \mathrm{Ag}_{\mathrm{n}, \mathrm{Cit}}{ }^{+}+\mathrm{Cit}(-\mathrm{H})^{\bullet} \rightarrow \\
& \mathrm{Ag}_{\mathrm{n}, \mathrm{Cit}}+\mathrm{DCA}+\mathrm{CO}_{2}\end{aligned}$ & & this work \\
\hline (15) & $\mathrm{Ag}_{\mathrm{Cit}}{ }^{+}+$wall $\stackrel{100^{\circ} \mathrm{C}}{\rightarrow} \mathrm{Ag}_{\mathrm{Cit}}{ }^{+}$-wall & & this work \\
\hline (16) & $\mathrm{Ag}_{\mathrm{Cit}}{ }^{+}$-wall $+\mathrm{Cit} \stackrel{{ }^{\circ} \mathrm{C}}{\rightarrow} \mathrm{Ag}^{0}$-wall $+\mathrm{Cit}(-\mathrm{H})^{\bullet}+\mathrm{H}^{+}$ & & this work \\
\hline
\end{tabular}

Table S3. Reduction reactions of gold ions in aqueous solutions. 


\begin{tabular}{|c|c|c|c|}
\hline & Reaction & $\begin{array}{c}\text { Rate constant } \\
\left(\mathbf{M}^{-1} \mathrm{~s}^{-1}\right)\end{array}$ & References \\
\hline (1) & $\mathrm{Au}^{\mathrm{III}} \mathrm{Cl}_{4}^{-}+\mathrm{e}_{\mathrm{aq}}^{-} \rightarrow \mathrm{Au}^{\mathrm{II}} \mathrm{Cl}_{3}^{-}$ & $5.7 \times 10^{9}$ & {$[48]$} \\
\hline$(2)$ & $\mathrm{Au}^{\mathrm{III}} \mathrm{Cl}_{4}{ }^{-}+\mathrm{H}^{\cdot} \rightarrow \mathrm{Au}^{\mathrm{II}} \mathrm{Cl}_{3}^{-}+\mathrm{H}^{+}$ & $5.7 \times 10^{9}$ & [48] \\
\hline (3) & $\begin{aligned} \mathrm{Au}^{\mathrm{II}} \mathrm{Cl}_{3}^{-}+\mathrm{Au}^{\mathrm{II}} \mathrm{Cl}_{3}^{-} \rightarrow & {\left[\mathrm{Au}^{\mathrm{II}} \mathrm{Cl}_{3}^{-}\right]_{2} \rightarrow } \\
& \mathrm{Au}^{\mathrm{I}} \mathrm{Cl}_{2}^{-}+\mathrm{Au}^{\mathrm{III}} \mathrm{Cl}_{4}^{-}\end{aligned}$ & $\begin{array}{l}2.9 \times 10^{8} \\
10^{2} \mathrm{~s}^{-1}\end{array}$ & [49], [50] \\
\hline (4) & $\begin{aligned} \mathrm{Au}^{\mathrm{I}} \mathrm{CN}_{2}^{-}+\mathrm{H}+\mathrm{H}^{+} \rightarrow & \mathrm{Au}^{0} \mathrm{CN}_{2} \mathrm{H}_{2} \rightarrow \\
& \mathrm{Au}^{0}+2 \mathrm{CN}^{-}+\mathrm{H}_{2}\end{aligned}$ & $\begin{array}{c}1.4 \times 10^{10} \\
5.3 \times 10^{4} \mathrm{~s}^{-1}\end{array}$ & [15] \\
\hline (5) & $\mathrm{Au}^{0}+\mathrm{Au}^{\mathrm{III}} \rightarrow \mathrm{Au}^{\mathrm{I}}+\mathrm{Au}^{\mathrm{II}}$ & & [50] \\
\hline (6) & $\begin{array}{c}100{ }^{\circ} \mathrm{C} \\
\mathrm{Au}^{\mathrm{III}} \mathrm{Cl}_{4}{ }^{-}+\mathrm{Cit} \rightarrow\left[\mathrm{Au}^{\mathrm{III}} \mathrm{Cl}_{3} \mathrm{Cit}^{-}+\mathrm{Cl}^{-} \rightarrow\right. \\
\mathrm{Au}^{\mathrm{II}} \mathrm{Cl}_{3}{ }^{-}+\mathrm{Cit}(-\mathrm{H})^{\circ}+\mathrm{H}^{+}+\mathrm{Cl}^{-}\end{array}$ & & {$[52]$} \\
\hline (7) & $\mathrm{Au}_{\mathrm{cit}}^{\mathrm{I}}+\mathrm{Cit}(-\mathrm{H})^{\bullet} \rightarrow \mathrm{Au}_{\mathrm{cit}^{\mathrm{I}}-\mathrm{Cit}(-\mathrm{H})^{\circ}}$ & & this work \\
\hline (8) & $\begin{aligned} \mathrm{Au}_{\text {cit }}^{\mathrm{I}} \mathrm{Cit}(-\mathrm{H})^{\bullet}+\mathrm{Au}_{\text {cit }}^{\mathrm{I}} \rightarrow \\
\mathrm{Au}_{2, \mathrm{cit}}^{+}+\mathrm{DCA}+\mathrm{CO}_{2} \\
\end{aligned}$ & & this work \\
\hline (9) & $\begin{aligned} & \mathrm{Au}_{2, \text { cit }}{ }^{+}+\mathrm{Cit}(-\mathrm{H})^{\bullet} \rightarrow \\
& \mathrm{Au}_{2, \mathrm{Cit}}+\mathrm{DCA}+\mathrm{CO}_{2}\end{aligned}$ & & this work \\
\hline (10) & $\mathrm{Au}_{2, \mathrm{Cit}} \rightarrow \cdots \cdots \rightarrow \mathrm{Au}_{\mathrm{NP}, \mathrm{Cit}}$ & & this work \\
\hline
\end{tabular}


a

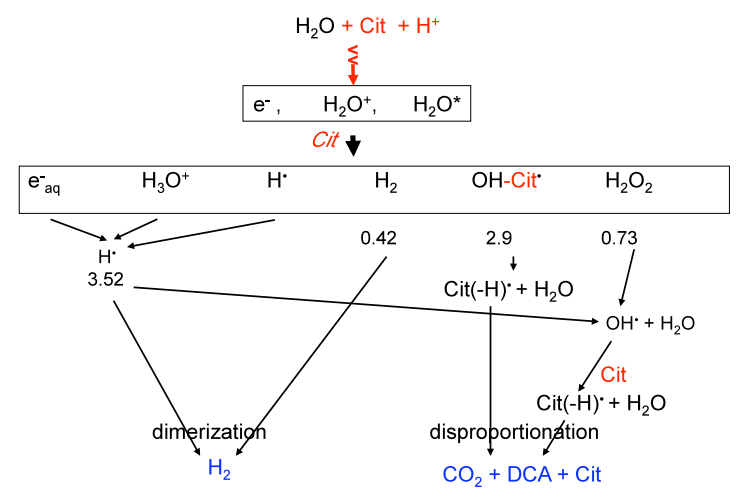

C

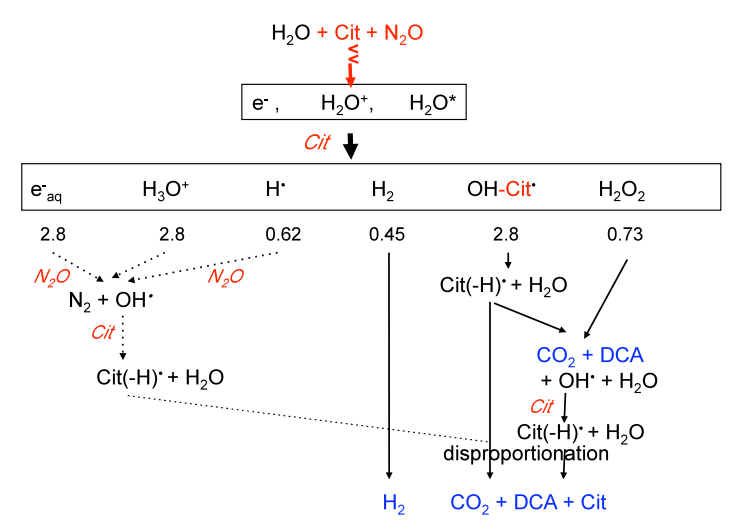

b

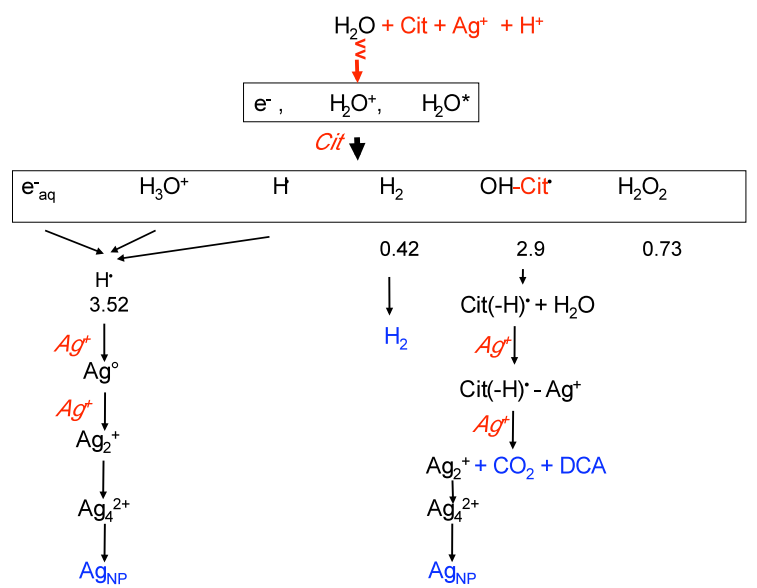

d

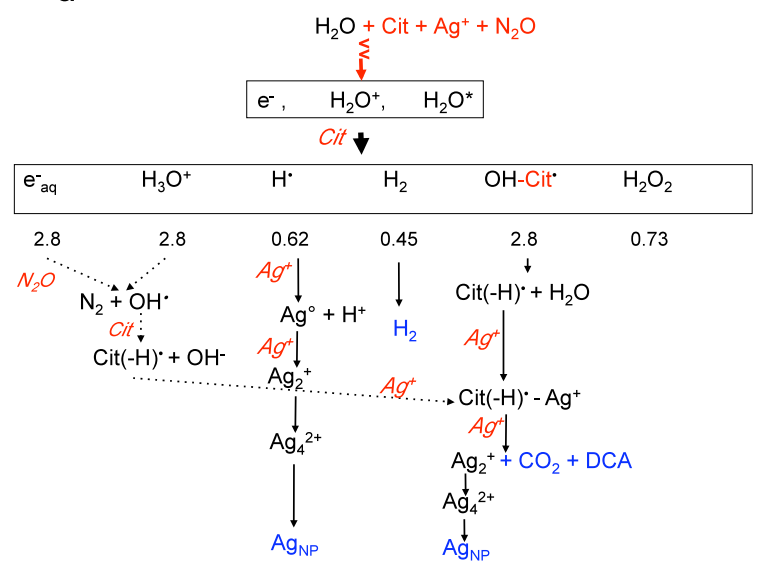

Figure S1. Scheme of the radiolytic oxidation mechanism of citrate ions without (a and $\mathbf{c}$ ) and with silver ions (b and $\mathbf{d}$ ) corresponding to the values of Table S1. The gases are measured only at $\mathrm{pH} 1.6$ and under argon (a and $\mathbf{b})$. But at $\mathrm{pH} 8$, the G(DCA) yield is equal to $\mathrm{G}\left(\mathrm{CO}_{2}\right)$. 


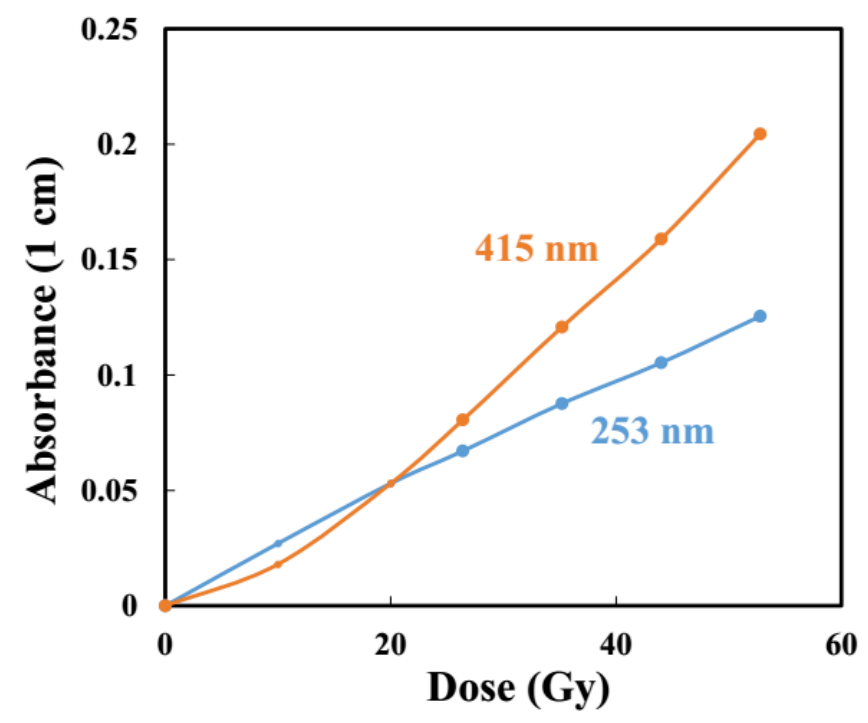

Figure S2. Optical absorption spectra of silver nanoparticles reduced by $\gamma$-irradiation at increasing absorbed dose at room temperature (gamma dose rate $8.8 \mathrm{~Gy} \mathrm{~min}^{-1}$ ) in $\mathrm{AgNO}_{3}(5 \times$ $\left.10^{-4} \mathrm{M}\right)$ and sodium citrate $(0.4 \mathrm{M})$ solution under $\mathrm{N}_{2} \mathrm{O}$ atmosphere. Reference: solution before irradiation. Optical path: $1 \mathrm{~cm}$.

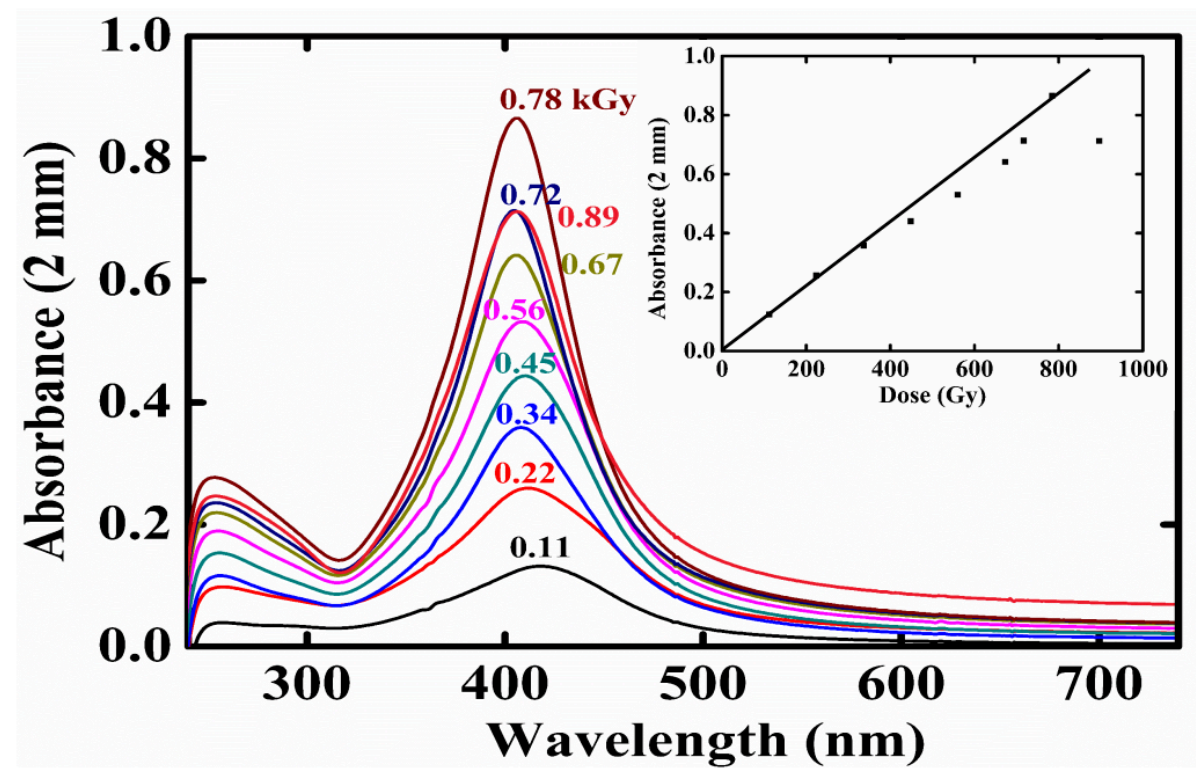

Figure S3. Optical absorption spectra of silver nanoparticles reduced by $\gamma$-irradiation at increasing absorbed dose (in $\mathrm{kGy}$ ) at room temperature (gamma dose rate $22.4 \mathrm{~Gy} \mathrm{~min}^{-1}$ ) in $\mathrm{AgClO}_{4}\left(5 \times 10^{-4} \mathrm{M}\right)$ and sodium citrate $(0.4 \mathrm{M})$ solution under $\mathrm{N}_{2} \mathrm{O}$ atmosphere. Reference: solution before irradiation. Optical path: $2 \mathrm{~mm}$. Inset: Dose dependence of the absorbance $\mathrm{A}_{405 \mathrm{~nm}}$ at the maximum of the plasmon band of silver nanoparticles $\mathrm{Ag}_{\mathrm{NP}}$. The yield $\mathrm{G}$ is deduced from the slope of the straight line. 


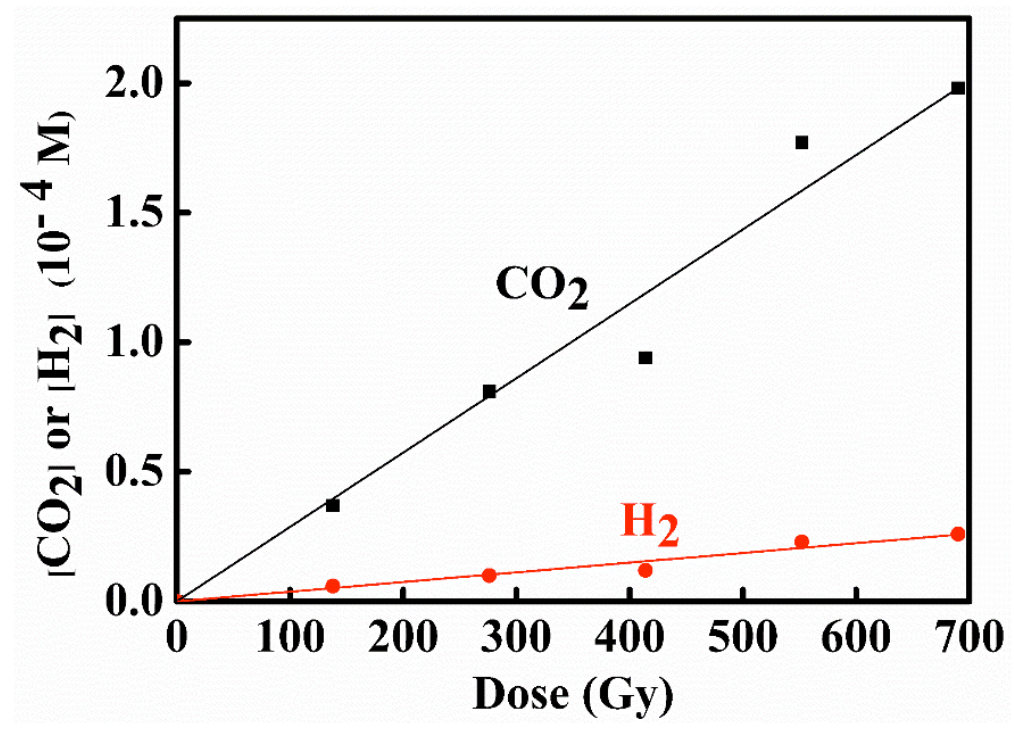

Figure S4. Dose-dependent concentrations of $\mathrm{H}_{2}$ and $\mathrm{CO}_{2}$ in Ar-saturated solution of $0.4 \mathrm{M}$ citric acid $(\mathrm{pH} 1.6)$ and $\mathrm{AgClO}_{4}\left(5 \times 10^{-4} \mathrm{M}\right)$. The yields are given by the slopes of the lines, $\mathrm{G}\left(\mathrm{H}_{2}\right)=0.37 \times 10^{-7}$ and $\mathrm{G}\left(\mathrm{CO}_{2}\right)=2.9 \times 10^{-7} \mathrm{~mol} \mathrm{~J}^{-1}$, respectively. The uncertainty is $\pm 5 \%$.

a

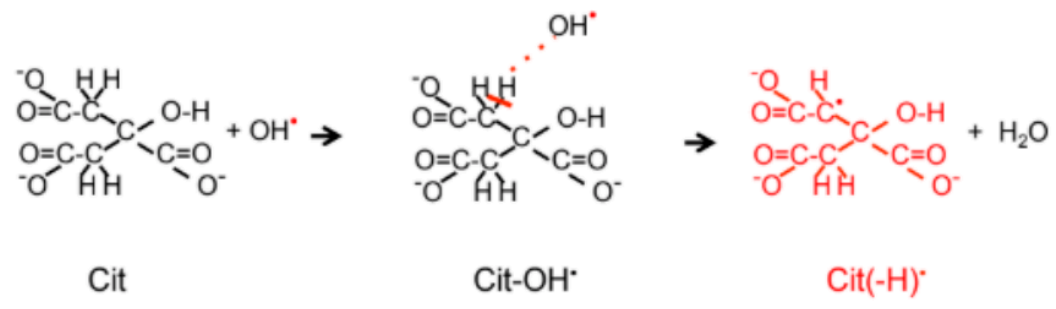

b

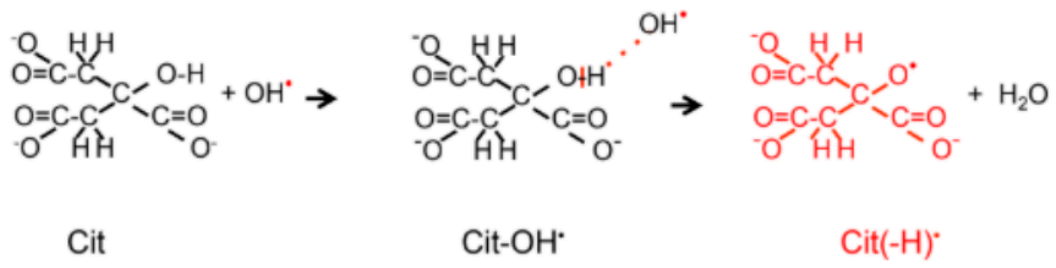

Figure S5. Schemes of the structures of citrate Cit, of the $\mathrm{OH}^{\circ}$-adduct and of the oxidized $\mathrm{Cit}(-\mathrm{H})^{\circ}$ radical. a. After attack on a $\beta-\mathrm{CH}_{2}$ - group. b. After attack on the hydroxy group. 


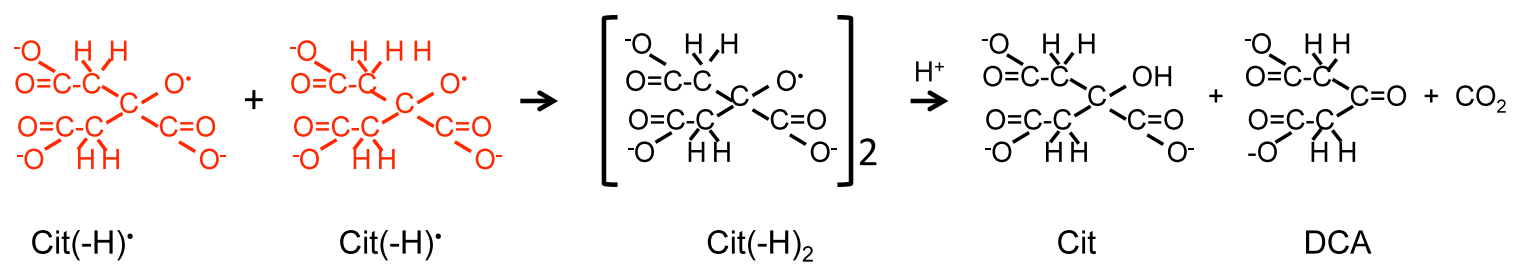

Figure S6. Scheme of the disproportionation of the radical $\mathrm{Cit}(-\mathrm{H})^{\bullet}$ into citrate and DCA and $\mathrm{CO}_{2}$.
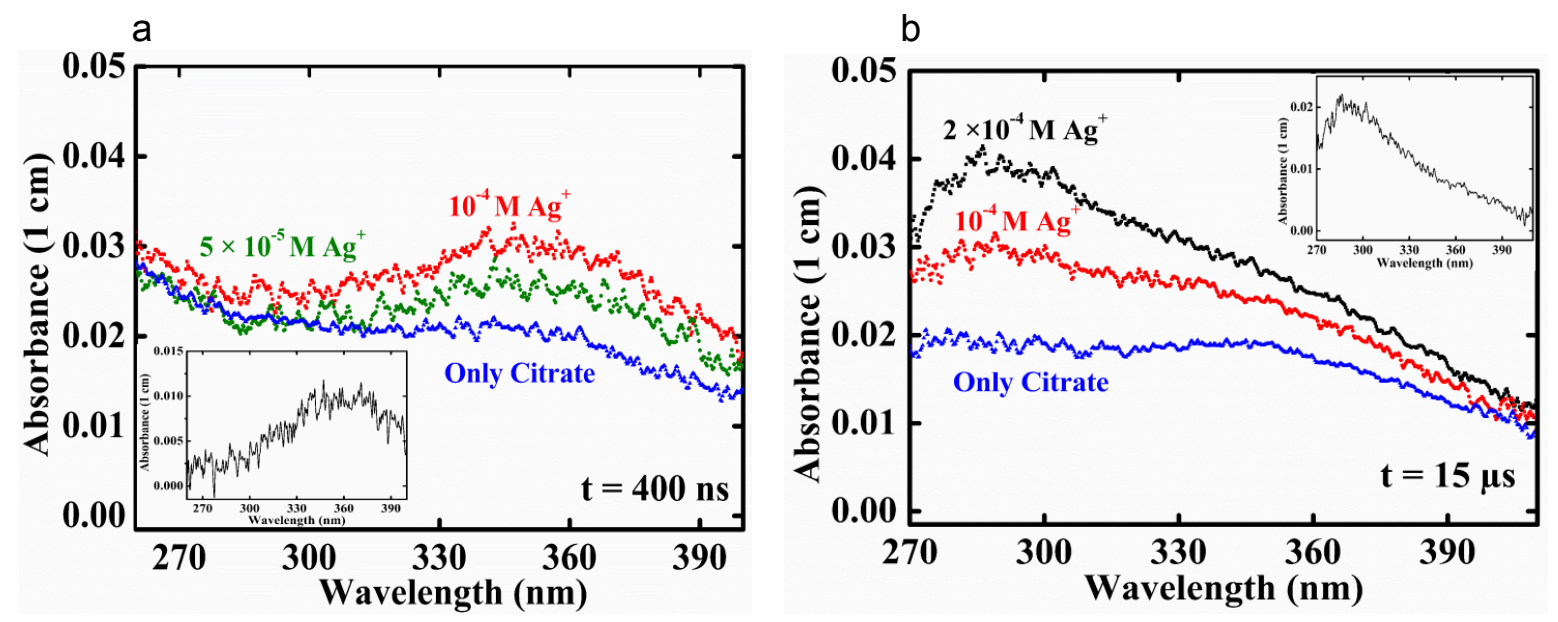

Figure 7. Transient spectra measured by pulse radiolysis of $\mathrm{N}_{2} \mathrm{O}$-saturated solutions of sodium citrate $(0.1 \mathrm{M})$ without and with silver perchlorate at various concentrations. Dose 35 Gy.. a. At 400 ns. Inset: Differential spectrum between the spectra of the $10^{-4} \mathrm{M}$ silver and free-silver solutions. b. At $15 \mu$ s. Inset: Differential spectrum between the spectra of the $2 \times$ $10^{-4} \mathrm{M}$ silver and silver-free solutions. 


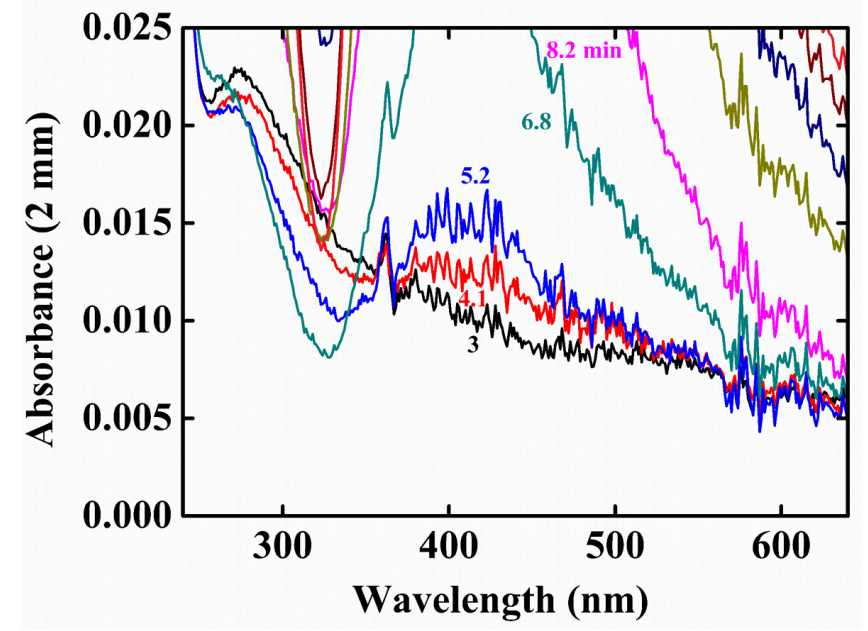

Figure S8. Transient absorption spectra of silver nanoparticles synthesized by the Turkevich method at the shortest times. $\mathrm{AgNO}_{3} 5.5 \times 10^{-4} \mathrm{M}$ and sodium citrate $4.4 \times 10^{-3} \mathrm{M} . \mathrm{T}=100$ ${ }^{\circ} \mathrm{C}$. Optical path $2 \mathrm{~mm}$ (same conditions as in Figure 6).

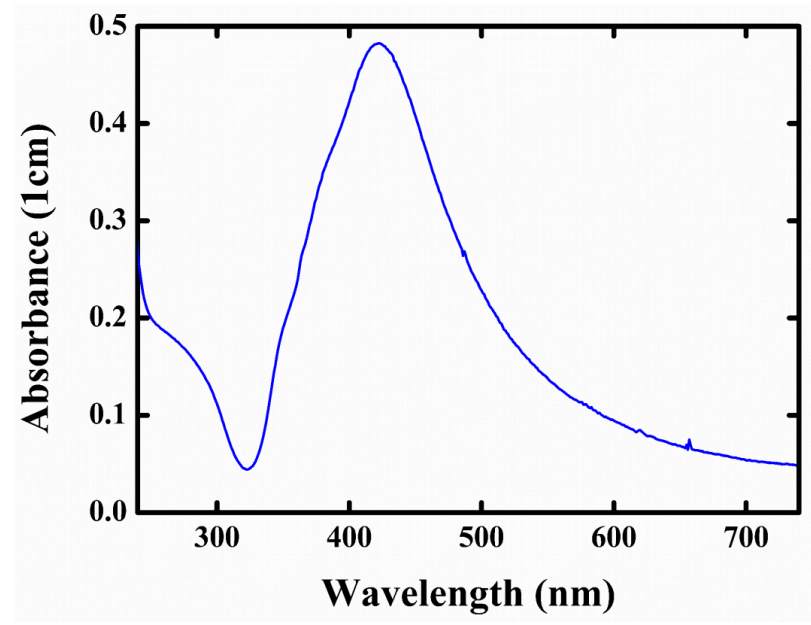

Figure S9. Absorption spectrum of silver nanoparticles prepared by the Turkevich method after 15 minutes at $100^{\circ} \mathrm{C}$ in $5.5 \times 10^{-4} \mathrm{M} \mathrm{AgClO}_{4}$ and $4.4 \times 10^{-3} \mathrm{M}$ sodium citrate. 